\title{
Optimization of the Enterprise Human Resource Management Information System Based on the Internet of Things
}

\author{
Haiqiu Li (iD \\ Department of Business Administration, Zhejiang Technical Institute of Economics, Hangzhou 310016, China \\ Correspondence should be addressed to Haiqiu Li; 260004@zjtie.edu.cn
}

Received 7 January 2021; Revised 23 February 2021; Accepted 26 February 2021; Published 8 March 2021

Academic Editor: Wei Wang

Copyright (c) 2021 Haiqiu Li. This is an open access article distributed under the Creative Commons Attribution License, which permits unrestricted use, distribution, and reproduction in any medium, provided the original work is properly cited.

\begin{abstract}
In this paper, the optimization of the enterprise HR information system is studied based on IoT first-off technology, the system demand phase is analysed, and the edge control system is designed and built. The hardware and software system and edge node management platform are implemented first, and then the communication scenarios between the edge layer of the system and the sensing layer, the edge layer, and the cloud layer are analysed, and the business type-driven link selection algorithm and the northbound multilink switching algorithm are designed and implemented, respectively, to guarantee the communication reliability between different layers of the system. Based on the implementation of the above functions, the edge control system can meet the intelligence, expandability, and security requirements of IoT applications. An in-depth investigation and research are launched mainly on the enterprise demand to determine the functional requirements and performance requirements of the enterprise and to achieve the basic logical structure; in the system design phase, the system architecture and other aspects of the design are realized. According to the conditions of the system function structure, a number of system module functions are designed in detail. The system is composed of the following modules, namely, personnel change management, organization management, and salary and benefits management. The system consists of the following modules, namely, personnel change management, organization management, compensation and benefits management, and personnel information management. The system modules run through the process of human resource management; in the system implementation stage, the system coding and page operation are realized based on the development tools and software development techniques. The system finally achieves the system design objectives and is put on a trial operation to meet its actual business requirements.
\end{abstract}

\section{Introduction}

Due to the advent of the information age and the rapid development of economic globalization, the market environment has also shown corresponding changes, and under the influence of this background condition, many enterprises have begun to realize that they should give full play to the value of human resources and turn them into their competitive advantage; however, to achieve this goal, not only must we build a sound human resource management strategy but also must use various techniques [1]. To achieve this goal, it is necessary not only to build a sound human resource management strategy but also to use various technical means to ensure the implementation of the human resources strategy [2]. With the development of computer and network communication technology, numerous devices are connected to the internet, the boundary between the real world and the virtual world tends to be blurred, and the internet is being extended to the physical world, forming the Internet of Things. In the IoT, the IoT search system is the key for computers to obtain information about the physical world and an important component to build a perfect IoT system [3]. IoT search refers to the use of relevant measures to obtain various information (e.g., objects, people, and web pages) from the IoT, store, and manage this spatial-temporal data information in an organized and orderly manner so that it can be easily searched by users [4]. The maturity of big data and $5 \mathrm{G}$ technology has completed the explosive growth of IoT business data, and this data volume is undoubtedly demanding in terms of computing power [5]. The architectural model of cloud computing has been relatively 
fixed, and even if there is a breakthrough in the hardware performance, it is difficult to complete the task efficiently, and only another efficient computing architecture and computing optimization scheme to assist cloud computing are the solution to cure the symptoms and the root cause [6]. Influenced by the relatively fixed model of cloud computing architecture, the network architecture serving the IoT also lacks some flexibility [7]. The future network should be adaptive to change with the change of business needs and environment, and this kind of intelligence is what is needed for IoT scenarios [8]. By improving the construction of HRM information technology, one can have a positive effect on the integration of management resources and thus reduce the expenditure of management costs. So far, there are numerous pieces of system software for HRM, but these system types are very homogeneous and are used for statistical data, with little interactivity as well as compatibility and more repetitive operations, which waste a lot of system resources [9]. With the emergence of integrated HRMS, management resources can be fully integrated to create favourable conditions for the development of various other tasks [10].

Lloret et al. explained that HRM informatization will bring great help to enterprise management, and they suggested that, in addition to storing HR data and payroll accounting, advanced management systems should have functions such as "labour costing," "data report generation," "monitoring recruitment," "tracking compensation and benefits," and "automatic attendance." [11] Professor Miao and his colleagues, through the study of the human resource information system, made it possible for the company to develop and implement a new system [12]. Seneviratne et al. analysed the relationship between information technology and competitive strategy, and based on the analysis of the competitive strategy and industrial structure, they discussed the strategic significance of HR information technology, only the successful use of modern information technology can enable companies to gain a competitive advantage and thus undermine their rivals in the competition, truly creating a new field of research on strategic information systems [13]. Pagan et al. studied on the implementation of HR information technology in a large number of enterprises, analysing the main difficulties encountered in the construction of HR information technology, proposing different schemes of process-oriented, model optimization and phased implementation, giving the objectives to be achieved by enterprises in different stages of development of HR information technology, and providing theoretical and example support for the construction of HR information technology in enterprises [14]. Kong et al. believe that the DBSCAN algorithm scans the whole data area, while each point is marked and set as the core object in the cluster, and the boundary objects located at the boundary of the cluster are set as noise, and then the number of objects within a certain distance around each object is determined [15]. The Dyer system is not only able to query static information of IoT entities but also to meet the specific needs of users to query the dynamic content information of physical entities in real time; it abstracts physical entities and sensors as web pages, which facilitates the search of physical entities and achieves seamless integration with traditional search engines [16]. The prediction mechanism is used by the Dyer system [17]. The prediction mechanism used by the Dyer system, while speeding up the efficiency of searching for spatialtemporal data information in the IoT, also reduces the precision of the query, allowing it to be applied in the IoT environment where system resources are more restricted [18]. However, these systems described above are usually singlefunction IoT search systems, which are not capable of handling multiple types of IoT spatial-temporal data simultaneously [19]. To realize a multifunctional IoT system, preprocessing and data classification of the collected IoT spatial-temporal data are required [20].

With the rapid improvement of the level of science and technology, we have ushered in the era of science and technology. The development and progress of science and technology have not only brought many conveniences to people's daily life but also provided a broader space for the development of enterprises. In the age of technology, increasingly new technologies have been applied to the daily management of enterprises, such as big data, Internet of Things, and other new technologies. The integration of new technologies and human resource management is also an inevitable trend for the future development of enterprises.

Therefore, how to make the effective combination of human resources and work efficiency; how to explore the maximum value of human resources; how to meet the needs of employees and, at the same time, achieve the goal of maximizing economic benefits; and how to find a suitable route for enterprise development in a complex market environment and timely response to human resources' problems are the primary issues that enterprises need to solve. In the current development of the society, due to the increasingly fierce competition in the market, coupled with the gradual improvement of electronic technology and information systems, under the influence of this background condition, the importance of human resource management is naturally highlighted. The human resource management information system has also been universally recognized by all sectors of society and has been widely used. In the future development of enterprises, the HR management system plays a very important role not only to adapt to the changes in the market environment but also as an important way to promote the reform process. With the system, the efficiency of personnel management is improved, while favourable conditions are created for the development of various tasks. Information security has become a hot topic in recent years, the internet, communications, and other industries are also increasingly concerned about the security of user information, and the data information handled by the human resources' management information system cannot be changed at will; in the future development, strict controls should be adopted to increase the protection of data to avoid theft of data resources by unruly elements and truly protect the personal information of employees. For enterprises, human resources are not only blood but also very important resources. 


\section{Analysis of Information System Design Based on No Internet of Things}

2.1. HR IoT Design. In our increasingly data-centric society, IoT systems have become an important component for generating spatial-temporal data. Various aspects of various fields, from environmental monitoring to smart manufacturing, cannot be separated from the IoT [21]. However, the key to build a complete IoT system is to solve the problem of huge data volume and spatial-temporal data classification of IoT. In the original cloud computing architecture, the user terminal (UE) or sensing terminal (SE) directly uploads the IoT data to the cloud, and the data are fed back from the cloud after the task is successfully executed, and the round-trip time delay of data transmission alone is not negligible [22]. Furthermore, the excessive concentration of data is a huge test for bandwidth, which causes network congestion and jitter and further affects the user experience of IoT applications. The introduction of edge computing architecture into the IoT system can effectively solve this problem. The edge control system model is to introduce the edge cloud part into the original cloud computing network system, which is composed of edge nodes and is the core of edge computing. The edge cloud is located inside the access network near the user side or understood as between the user layer and the access network. It is because of this spatially distributed deployment feature that the edge control system has lower task processing latency and more customizable system functions. The scenario model diagram of the edge control system introduced in this paper is shown in Figure 1.

The static-priority scheduling algorithm is to give different business processes different priorities, and this priority will not change during the system operation, i.e., the system always executes the business process with higher priority. In the actual environment, the low-priority regular monitoring business occurs more frequently, so the system runs this business process most of the time [23]. High-priority services such as emergency alarms occur less frequently, but once they occur, the system will give up the processing of the other three types of services and give the CPU processing power to the alarm business processes. Preemptive scheduling means that the system strictly follows the priority of the business to process business processes. When a high priority arrives, the system prioritizes all current tasks and starts execution from the highest priority task.

To get the optimal offloading policy, the input of the algorithm should contain information about the demand for computation and storage resources of all subtasks in the task set. Also, if it is currently the peak period of task generation, then NEN is likely to have no remaining to compute or storage resources. Therefore, when pressing subtask information into the task queue, the subtasks that take longer to execute can be pressed into the task queue first, and these subtasks will also be prioritized when subtasks are offloaded. This strategy ensures that the execution time of the original task $T$ is reduced as much as possible when there is a peak in task generation. The subtask factors that need to be considered for multiedge node computation collaboration include the expected execution time of subtasks PT, the computational resource requirements of subtasks PC, and the storage resource requirements of subtasks PS. Since the number of CPU cores in NEN is limited and PC is related to PT, PC of subtasks can be set to 1 core and 2 cores according to the relationship between $\mathrm{PT}$ and the average expected execution time age of subtasks in TS.

$$
\begin{aligned}
M_{i} & =\frac{1}{N} \sum_{n=1}^{N} \sum_{j=1}^{N} M_{j}^{T} M_{i}^{2}\left(T_{n}\right), \\
M_{i}^{2}\left(T_{n}\right) & =\sum_{n=1}^{N} \sum_{i, j=1}^{N} T_{i}^{j} I_{i}^{2} .
\end{aligned}
$$

The organization management system is the basic module of the HR system, and the main responsibility is to manage the company's subordinate departments, including information about the organization at all levels, staffing, and management of staff employment, to facilitate HR managers to implement comprehensive management, and to provide statistical charts of all departments' employment and staffing to facilitate the general manager to understand the organization information and to have an in-depth understanding of staff employment to make more scientific decisions. The main functional nodes of organization management are shown in Figure 2.

According to these two preprocessing steps, subtasks with larger expected execution time will be offloaded first and will be offloaded to the NEN with the smallest required synchronization time, taking into account the balanced relationship between the expected execution time PT of subtasks and the synchronization time dT of subtask files.

$$
U_{5 * 4}=\left[\begin{array}{cccc}
1 & 1 & 0 & 1 \\
1 & 0 & 1 & 0 \\
0 & 1 & 1 & 1 \\
1 & 0 & 1 & 0 \\
1 & 1 & 1 & 1
\end{array}\right] .
$$

The algorithm has a good classification for the data transmitted in real time by IoT mainly for cleaning and classifying the transient abnormal data collected by IoT nodes with normal data. And when there is persistent damage to sensor nodes, network disconnection, etc., the algorithm will not be able to achieve the processing of spatial-temporal data. However, in the IoT real-time search system, the system sets up a sensor node and network anomaly monitoring module. When there is an abnormality, the system will get feedback to facilitate the timely maintenance and management of sensor nodes.

As the IoT real-time search system, the collected data are continuous, and usually, not much variation is found between adjacent numbers. 


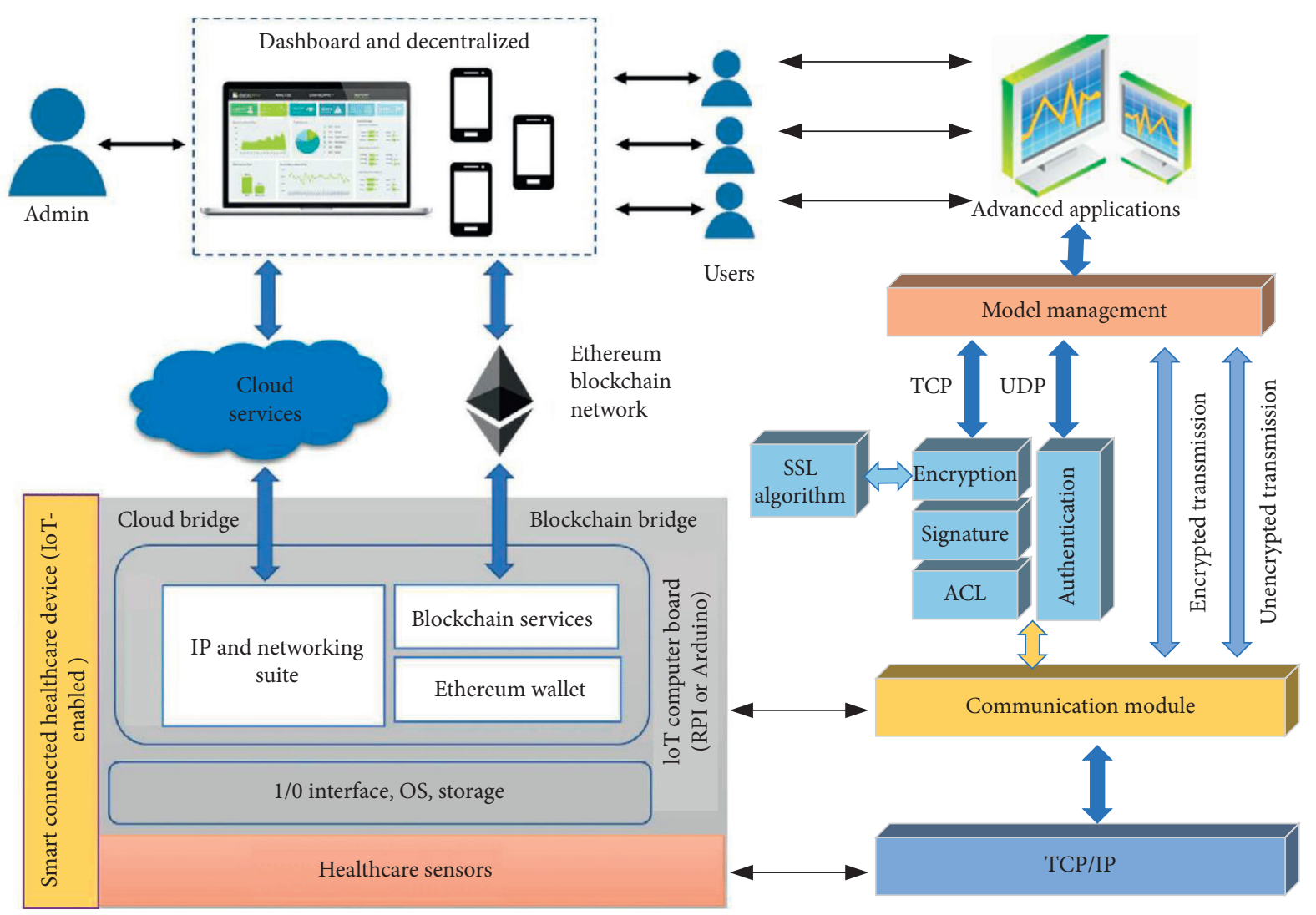

FIgURE 1: HR IoT framework.

$$
\begin{gathered}
G_{j}=\frac{\sum_{i, j=1}^{m, n} R_{i}^{j} * U_{i j}^{j}}{\sum_{i, j=1}^{m, n} E_{i} * U_{i j}}, \\
F(x)=\left\{\begin{array}{l}
C^{2} r(x), 0 \leq x \leq 0.6, \\
S^{2} r(x), 0.6<x \leq 0.9, \\
N^{2} r(x), 0.9<x \leq 1 .
\end{array}\right.
\end{gathered}
$$

For the characteristics of the real-time search system of IoT based on spatial-temporal data, the classification network should not only meet the speed that can handle IoT classification but also needs to meet the classification accuracy with good algorithm robustness that can quickly handle abnormal objects and data.

$$
\operatorname{tab}_{R}(i)=r\left(\left(G_{\text {mean }}^{2}+1+M_{i}^{2}-G_{\text {mean-2 }}^{2}\right) \times \operatorname{cdf}_{R}^{2}(i)\right) .
$$

The IoT nodes can be controlled quickly and can achieve real-time feedback and operation of the collected data without supervisory interference. For example, when the system detects an oil leak, the pump will be automatically shut down; when the system monitors a change in wind speed, the direction of the wind turbine will change; and when the IoT system detects a high concentration of hydrogen sulphide in a dairy farm, it will automatically alarm and promptly notify the person in charge of the safety management of the dairy farm and the relevant safety production management department [24].
2.2. Information System Design. The system construction contains 29 different functional modules, including basic modules and extension modules. There are 17 different modules in the basic module, including organization management, contract management, personnel change management, personnel information management, and salary management. There are 12 different modules in the extension module, including party management, performance management, professional talent management, and decision analysis. In this article, we mainly design the modules of organization management, salary management, personnel change management, and personnel information management in detail, as shown in Figure 3.

The organization management system is the basic module of the HR system, and the main responsibility is to manage the company's subordinate departments, including information about the organization at all levels, staffing, and management of staff employment, to facilitate HR managers to implement comprehensive management, and to provide statistical charts of all departments' employment and staffing to facilitate the general manager to understand the organization information and to have an in-depth understanding of staff employment to make more scientific decisions [25]. The personnel change management module is mainly responsible for managing the change information of staff categories, positions, and duties. Also, it is mainly responsible for separation, redeployment, and conversion, covering the following types of changes: intracompany redeployment, cross-company redeployment, resignation, dismissal, 


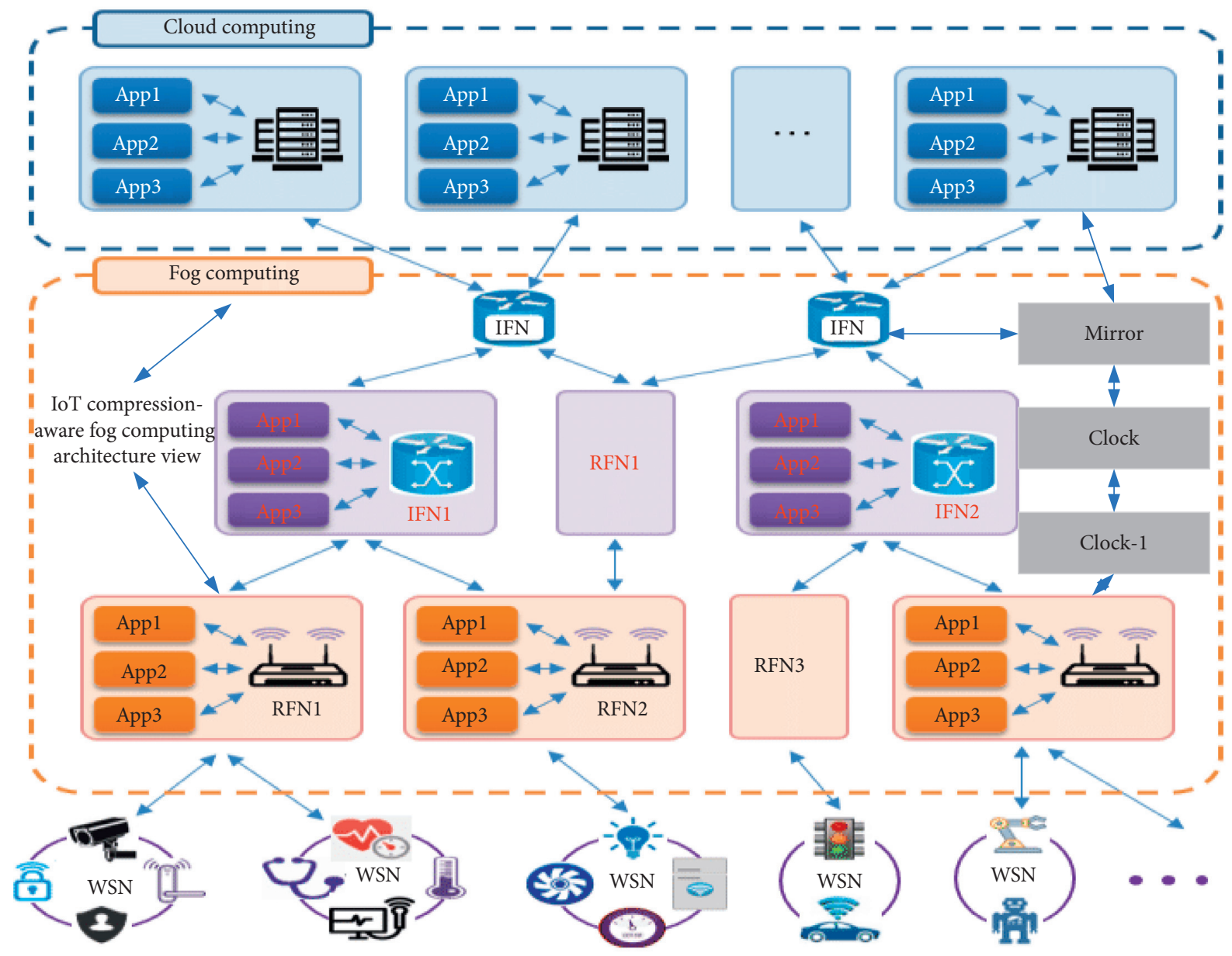

Figure 2: Decision model diagram.

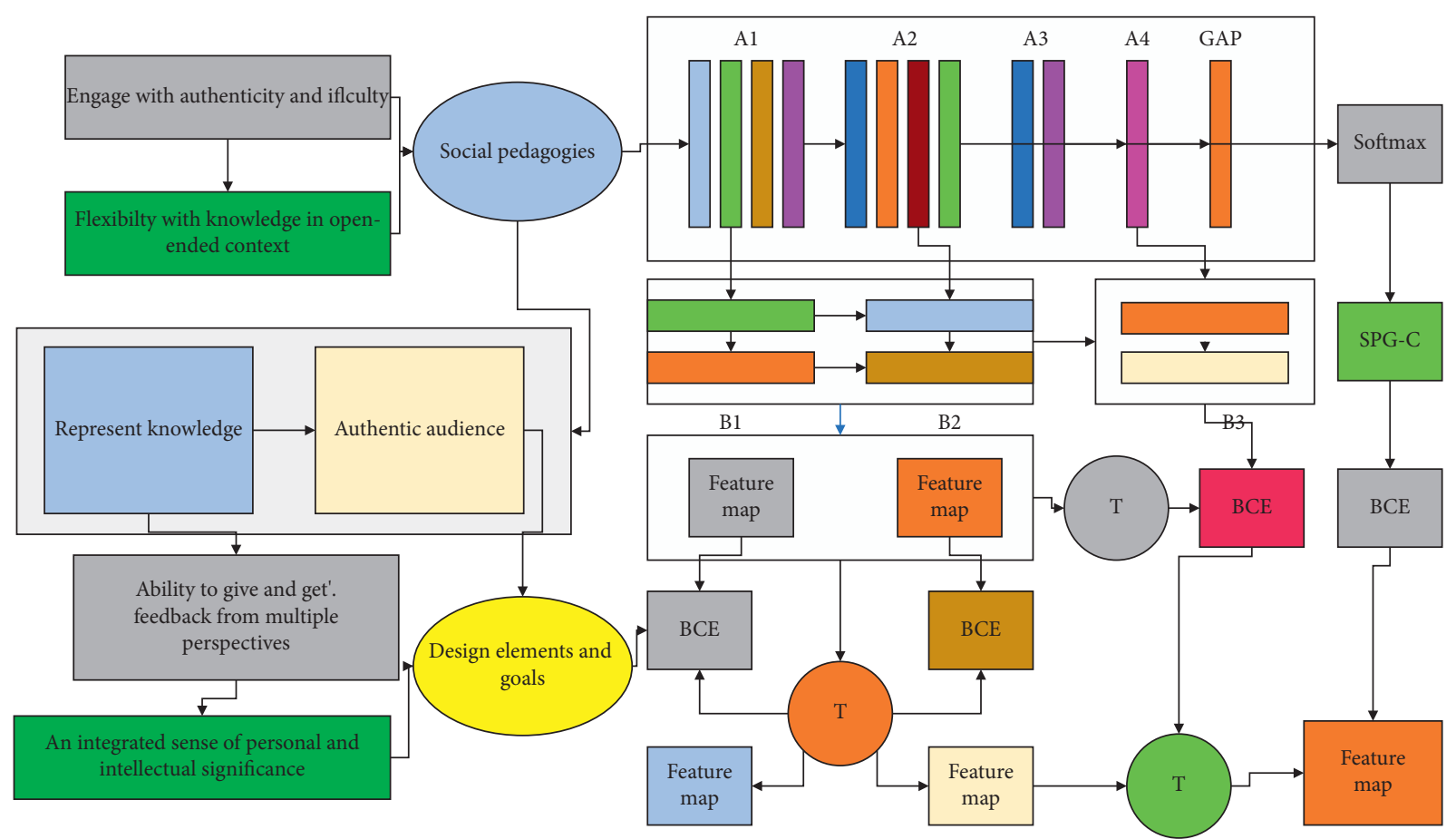

Figure 3: Overall system module. 
termination, negotiated termination, layoff, retirement, leave, death, and extended retirement. For staff transfer business, it allows two application methods: approval process and direct approval; for staff deployment and staff separation business, it allows four application methods: no approval, direct approval, approval process, and direct record in deployment record/separation record. After all the conversion operations are processed, relevant information will be entered into the personnel information, such as the date of conversion and whether to convert. After the implementation of the redeployment process, the relevant information is also entered into the personnel's employment status, such as employment start, personnel category, and redeployment type; it is also possible to directly generate a transfer request form. After the implementation of separation, the same information will also be entered into the separation of personnel, such as the date of separation and the type of personnel after separation; and in the process of separation, because the contract is still valid and there is an employee agreement, they will also be reminded. The functional design profile is shown in Table 1.

The database design mainly includes three stages of work: requirement analysis of the database, design of the logical structure of the database, and design of the physical structure of the database. The invoicing system directly shares data and generates invoice data; at the same time, the statistical table of the Suyuan charging system generates a detailed table of charges, which are directly imported into the financial system to generate corresponding vouchers; through the form settings of the financial system, the generation of the budget execution table is increased, and the comparison table between financial data and budget data for the corresponding period is directly generated to improve the efficiency of financial personnel in tracking and controlling the budget execution. During the trial run, a feedback seminar was held regularly. The meeting was chaired by the leader of the IT optimization team, and all members of the IT optimization team participated in the meeting.

2.3. System Optimization Design. This paper mainly researches and proposes data classification and abnormal data processing algorithms that can be performed on the spatialtemporal data collected by the IoT. Real-time classification of the collected data and distributed real-time storage of the collected spatial-temporal data using a temporal database can increase the fast search capability of the IoT system so that the IoT search system can search multiple types of IoT data at the same time; and the target tracking algorithm for spatial-temporal data is studied, which improves the efficiency and accuracy of target tracking [26-29]. The research content of this paper mainly contains the following four points: study the existing deep learning algorithms, analyse how to use deep learning algorithms and IoT real-time search system for effective combination, face the diversity of search data in the IoT search system, and study the use of deep learning algorithms for data cleaning, data dimensionality reduction, data feature extraction, and semantic understanding of IoT real-time transmitted data. Then, the deep learning algorithm is used to classify the real-time transmission data of IoT and design a reasonable spatial-temporal data storage structure to realize the chunk storage of real-time spatial-temporal data collected from IoT, which accelerates the indexing speed of the data.

The target tracking algorithm based on IoT spatial-temporal data is designed. The existing target tracking algorithms and convolutional neural network algorithms are studied, how to use target tracking algorithms for real-time dynamic target tracking is analysed, and deep learning algorithms are used to quickly achieve target identification and improve the accuracy of target tracking. The platform of the IoT search system is designed, the cloud architecture for the IoT search system is proposed, the data storage structure for spatial-temporal data processing is designed, the system efficiency of IoT real-time search is accelerated, and finally, an IoT search system is realized. The implemented system has the functions of the realtime query of location information, temperature and humidity information, gas concentration information within the specified range, and realization of camera-based target tracking, face detection, face recognition, etc., as shown in Figure 4.

Due to the target tracking-oriented IoT search and monitoring system, the collected data have the nature of complexity, real time, large data volume, etc. At the same time, the search and monitoring system needs to meet the requirements of system query and real-time performance. At this time, the traditional server structure alone cannot meet the performance requirements, and the server memory is prone to be insufficient and the server load is too large. Therefore, we need to build a spatial-temporal data processing platform as shown in Figure 4 to process the real-time data collected from the Internet of Things and provide data storage services for the Internet of Things search system. The spatial-temporal data infrastructure layer provides the operating environment for the normal operation of the spatial-temporal data service layer and spatial-temporal data application layer, including computing facilities, storage facilities, and network facilities. The spatial-temporal data service layer provides various data services for IoT search information systems and industrial application systems, including data exchange and sharing services, data cleansing, integration services, and data analysis and mining services. These data services can also be used by the application layer of the spatial-temporal data in the upper layer. The service gateway provides a unified access point for users outside the spatial-temporal data centre to access various services and applications provided by the spatial-temporal data centre and carries out the necessary authority management. Here, users outside the spatial-temporal data centre include government departments, police, and the public. The users can access various services and applications provided by the spatial-temporal data management centre through the IoT search system for target tracking, face recognition, environmental monitoring system, and internet applications, respectively.

In the experiment, the same temperature sensor is put into the refrigerator for some time, and the data collected by the sensor will be displayed on the front of the web page; 
TABLE 1: Functional design profile.

\begin{tabular}{lccc}
\hline Primary node & Secondary node & Introduction to functional design & Values \\
\hline Basic settings & 1 & Basic crotch case & 12.518 \\
Basic settings & 2 & Salary period & 3.154 \\
Basic settings & 3 & Salary standard category & 45.257 \\
Basic settings & 4 & Salary standard table & 14.879 \\
Basic settings & 5 & Salary standard setting & 46.578 \\
\hline
\end{tabular}

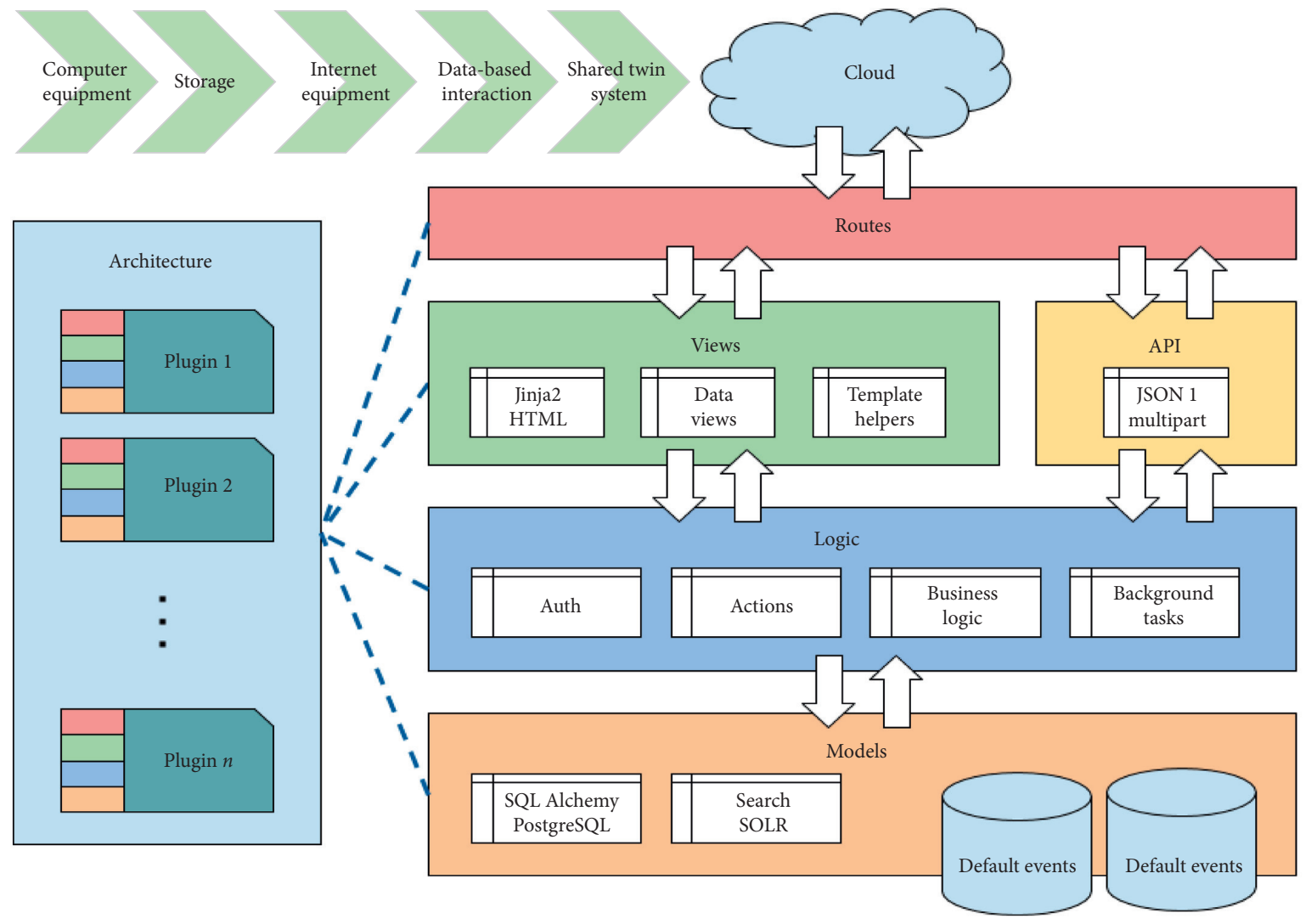

FIgURE 4: Optimized design framework.

the real-time temperature of the refrigerator fresh layer, where the vertical coordinate represents the temperature data and the horizontal coordinate represents the time, is the same as the actual temperature of the refrigerator measured by using the physical thermometer. During the experiment, as the sensor is enclosed inside the refrigerator, there may be slight fluctuations in the data due to the instability of the signal situation. Support automatic data processing of salary standard changes caused by salary system reform, salary standard adjustment and grading, promotion, advancement, and grade promotion. It supports fixed salary adjustment business. When employees face job changes such as transfers and promotions, the system will automatically adjust the salary ratio. Support the establishment of salary, insurance, and benefit accounting rules in the system, and automatically calculate the corresponding results according to these rules.

A real-time IoT search system is built, and the system can be through a variety of sensors including RFID, cameras, temperature and humidity sensors, MQ136, and other devices for information collection and information through the encrypted, secure, and stable transmission server backend; the front-end personnel can query the required IoT information through this real-time IoT search system while building an Android client based on face target recognition. Through the tracking of the person's photo information required by the client upload, the system background will automatically match the facial information captured by the camera in the most recent period of time and return the latest camera position, geographic location, and other information about the person to be captured. This can retrieve customers to facilitate the pursuit and discovery of goals. The main page of the IoT search system is based on spatial-temporal data, and the system mainly contains a real-time video monitoring module, a face-based target-tracking query module, and a search module.

\section{Analysis of Results}

3.1. System Performance Results. In the construction of comprehensive departmental information, the departmental 
file node is the foundation, which can also provide data support for human resource management and can also share data with other related management, helping to reduce its basic input volume. The function of job management is to better maintain the relevant information in the job system and realize the operation of deleting, adding, editing, and modifying job information. In this node, each unit can obtain the corresponding organization chart according to its management scope and its viewing habits. Here, one can freely set the orientation, background colour, connecting lines, fonts, display items, and borders of the organization chart and select the positions or departments to be displayed according to one's needs. In addition to the clear hierarchy of the company, one can also click to check the personnel or positions under the hierarchy and browse the detailed information of the personnel, including their names and photos. One can also print the cards of the employees directly. The work of post management is mainly to build the system of group posts, specifically including the maintenance of post creation and the selection of post successors; also, it should be combined with the actual posting and establishment of personnel, browse the historical posting information and the current posting information, and analyse the posting situation of the post or department, as shown in Figure 5. Job information management work is mainly to set the following job-related information: job quality indicators, job basic information, job work authority, and job qualifications, and one can browse, download, and upload job-related attachments here and support the output of job list and job description. Job posting browsing mainly analyses the posting situation, provides the function of browsing the current posting personnel and the former posting personnel, which makes it possible to query the posting history posting information.

Personnel information maintenance is used to maintain personnel information, where the personnel information mainly covers personal information, subset information, and job information. You can also add users, personnel card, and personnel roster for joint inspection and support batch personnel card and personnel photo export operations, such as enabling the ability quality management module. This can apply the ability matching function to view employees and work ability qualities. If the competency management module is enabled, one can apply the ability matching function to view the matching situation and analysis results of employees and positions, as shown in Figure 6.

Statistical analysis is mainly used for analysis of employees' work information, personal information, and their information, including current employees, transferred employees, terminated employees, and retired employees. Flexible statistical analysis of employee information can be performed, and the results of statistical analysis can be displayed through a variety of graphics. Based on the information of deployment records, the flexible statistical analysis of employee deployment can be realized, and statistical data and various statistical analysis graphs can be provided. The salary system is built based on positions or posts and oriented to the performance, and at the same time, it should consider fairness and efficiency. e-HR system's

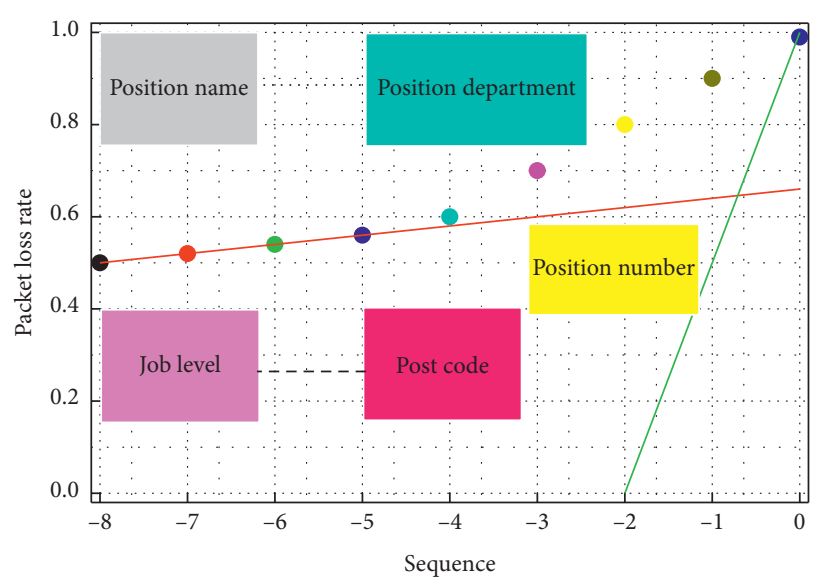

FIgURE 5: Job information statistics.

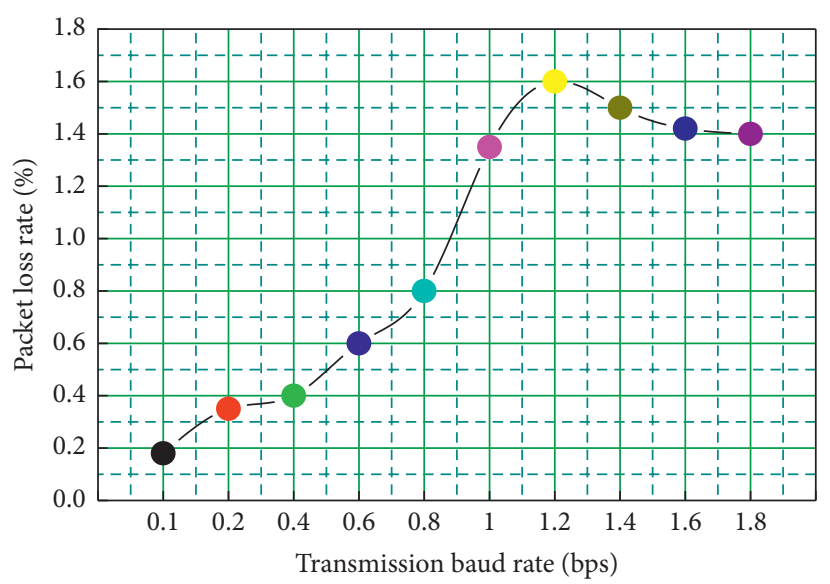

Figure 6: Personnel information query.

salary system construction is mainly the information realization of the enterprise salary system, including the establishment of the multi-payroll category scheme, the configuration of the salary rule table, salary item setting, salary period, tax rate table, substitute bank configuration, and statistical report. On this interface, one can set various attributes of payroll items, including the rules of taking numbers, whether to include in the payroll system, whether to carry over to the next period, and whether to count tax items. After the items are configured, subordinate units can directly refer to them, as shown in Figure 7.

In this interface, one can set the payroll period and refer to the accounting period so that one can generate the natural monthly payroll period and configure it directly from the actual needs, and in this interface, one can configure the issuance category of the unit's payroll items and directly refer to the public payroll items so that one can realize the personalized configuration of the number taken, and one can import the data used for payroll accounting outside the system. After the system was successfully tested, the system was applied comprehensively and achieved good results, especially the query engine which can be flexibly set and statistical output, which has changed the way of manual statistics in the past. 


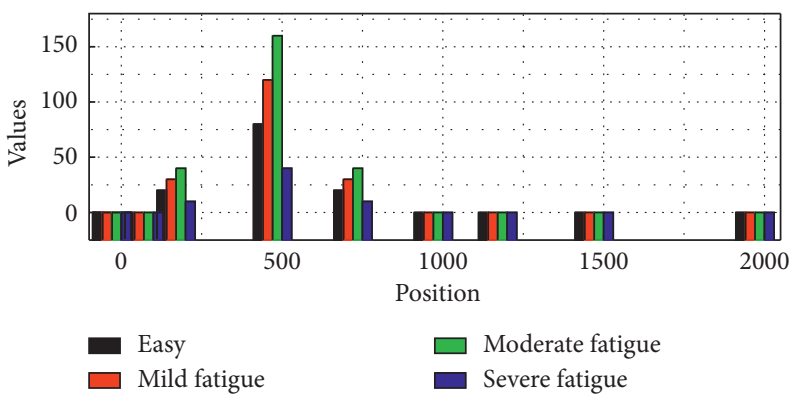

(a)

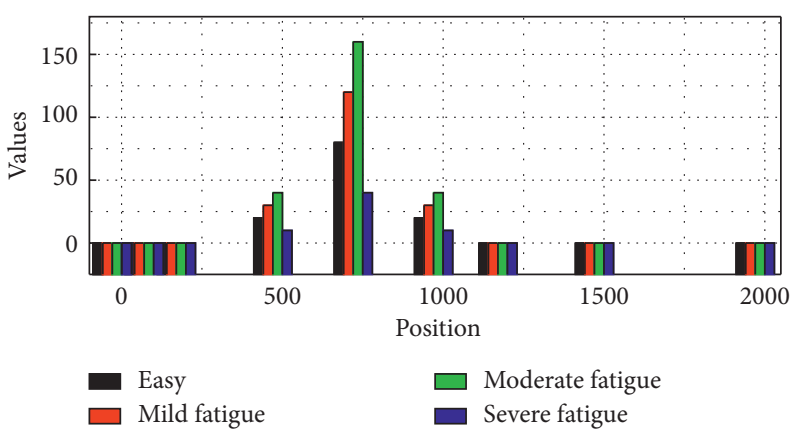

(b)

Figure 7: Payroll statistics.

The realization of organization management module, personnel information management module, employee change management module, and salary management module provides many accurate data supports for scientific decision-making. The system running environment and installation requirements are introduced, and the system's functions are tested by the black-box testing method. The results show that the information system can meet the needs of the company's human resource management.

\subsection{Analysis of Optimization Results. When planning in-} formation technology systems for service companies, not only administrative and financial management systems are involved but also business management systems, which are like those of most companies in other industries. In addition to the company's point of view, we need to consider how to optimize the management of business quality through the information system, how to strengthen the management of personnel scheduling to save costs, how to strengthen the interaction with customers, etc. We also need to consider how to effectively manage the facilities and equipment from the perspective of the project customers to extend the service life and thus realize the value of the property and how to strengthen the parking management to manage the public revenue of the project customers and ultimately maintain the interests of customers. Because of the above needs, as the manager of the company's business management department held several seminars and after many discussions with information technology professionals and business personnel, the optimization of the information technology system was planned as a whole, from different management perspectives and different business modules, as shown in Figure 8.

The original OA office system is upgraded. Firstly, the approval process is further sorted out so that the control authority of the company's work is implemented into the system more intuitively and concisely; secondly, the material management is strengthened, and in the process of approval of the material application, the inlet and outlet management of the material is carried out simultaneously, and the use of the material is tracked and managed to avoid unnecessary waste and improve the control ability of cost; thirdly, the original machine attendance is upgraded to the company, and the original machine attendance is upgraded to software attendance, which can remotely supervise the attendance of personnel in each project management office through the cell phone positioning function and generate attendance sheets by monthly summary calculation. In addition to the changes, the company is also considering to implement a salary management system, asset management system, and warehouse management system one after another and is actively carrying out demand statistics and market research work. If payroll, asset, and warehouse management can also be integrated into the $\mathrm{OA}$ office system, the payroll can directly capture the attendance results to ensure the accuracy of calculation; the depreciation of various assets stored in the warehouse or the warehouse inventory can also become the reference basis for initiating the requisition process, and the company's assets and low-value consumables can be jointly controlled at the front and back ends to avoid waste and save costs, as shown in Figure 9.

The service system includes different functional modules such as an on-site quality verification system, maintenance intelligent scheduling system, facility and equipment inspection and maintenance system, and customer relationship management (CRM) system. Through these different functional modules, we can realize the management and dispatching of service personnel within the project scope, the inspection management and maintenance management of equipment, and the full supervision and management of service process, service speed, and service effect by all management levels of the company, so as to realize the performance management of the organization; through the customer relationship management (CRM) system, we can establish the customer service centre of $\mathrm{T}$ company and realize the management through the CRM system; T company's customer service centre can register, assign, track, and give feedback to customers' complaints, suggestions, and repair reports in a "total-sub-total" manner, thus achieving the purpose of improving the motivation of employees, improving service quality, enhancing the service level, and saving operation cost; through the overall linkage of each module system in technology, the workload of employees, service quality, service efficiency, and other related data can be tracked. Quantitative statistical forms can be formed to facilitate the data collection of quantitative indicators in the performance assessment of employees; 


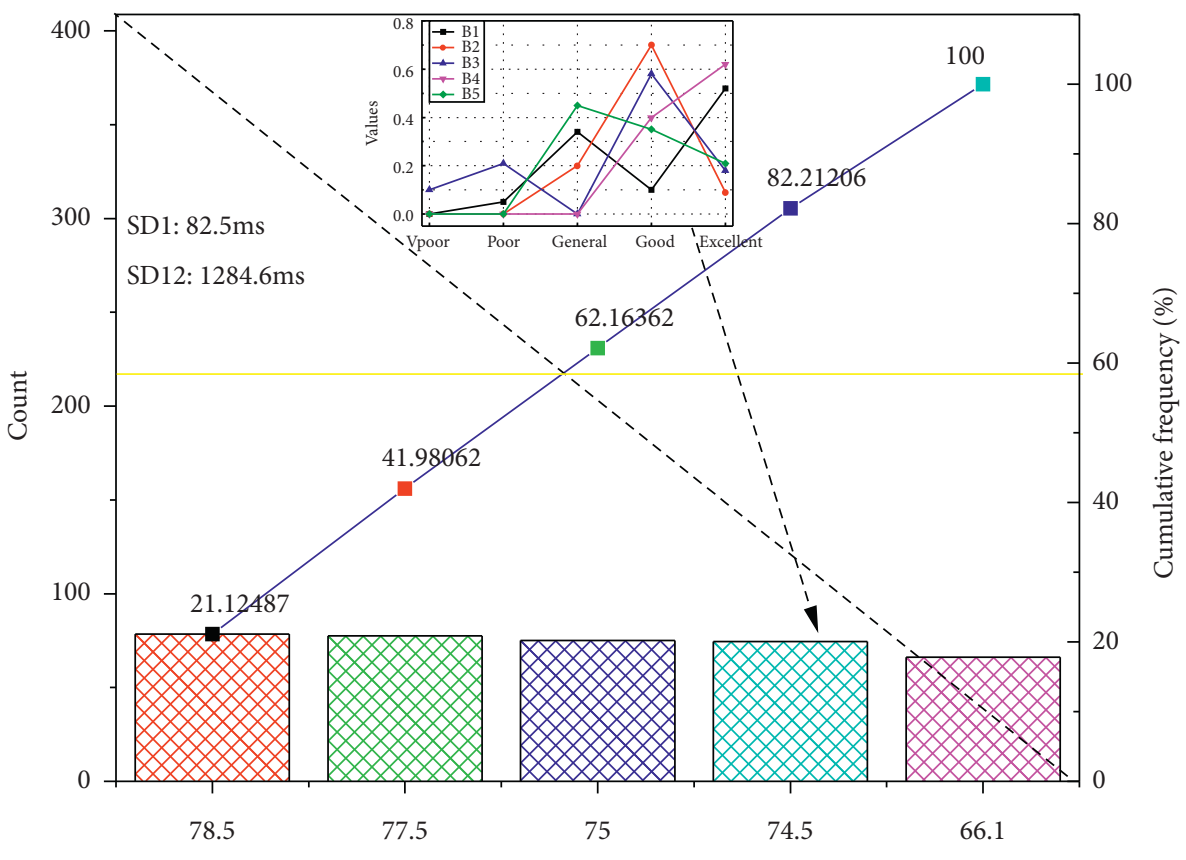

FIgURe 8: Average processing time.

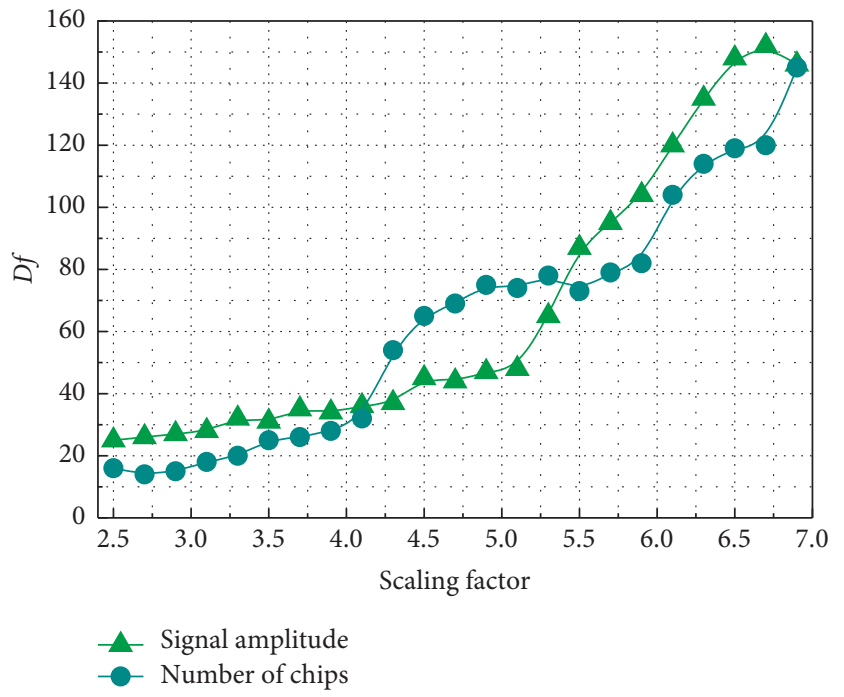

FIgure 9: Total Cost Analysis.

through the maintenance intelligent dispatching system, employees can be dispatched statistically and efficiently, and the workload of employees can be counted through dispatching records and statistical forms and directly linked to their salaries, as shown in Figure 10.

As the person in charge of the leading department of information technology optimization, information technology professionals are required to negotiate and communicate with information technology system suppliers, and in the process of optimizing the implementation of information technology systems in the above areas, attention has to be paid to the diversity and compatibility of the ports of communication with system suppliers. To ensure data

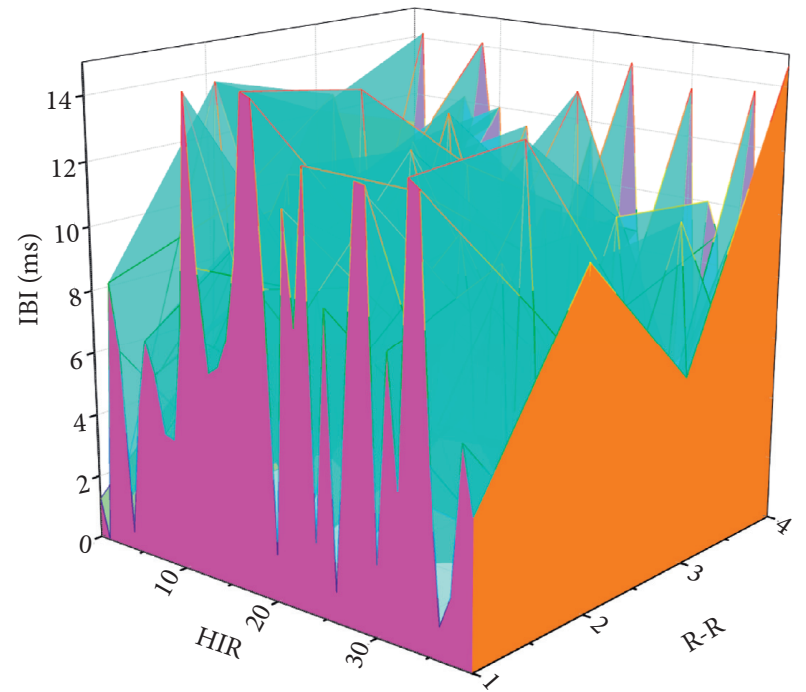

FIgURE 10: Manpower cost analysis interface diagram.

sharing and resource sharing between different functional systems and to meet the import of data forms, the relevant data information should be synchronized with the charging information of the Suyuan management system; the information of the attendance management system should be imported into the salary management system to meet the demand of salary calculation; the information of the customer relationship management (CRM) system should be consistent with the information of the maintenance intelligent dispatching system and the follow-up information of task implementation in the site quality verification system. The system should be consistent and eventually form a closed-loop service. Since various information systems are 
more professional, the promotion speed of each information system must be determined according to the management priority of the company. In order to give full play to the integration of information technology management information, it can be realized by building a data platform and data mining. The integration and sharing of information and resources in each link of the service process can enhance its market competitiveness.

\section{Conclusion}

A centralized multitasking scheduling method with a single edge node is proposed to address the problem of how to reduce the processing latency of small tasks in IoT scenarios. The method first designs a multilevel scheduling framework combining process scheduling and thread scheduling, and the process scheduling uses a preemptive static priority scheduling algorithm for different kinds of services. The thread scheduling targets the same kind of business with high concurrency, and this paper proposes a task execution urgency factor and designs a dynamic priority-based thread scheduling algorithm, where the urgency factor is set by the remaining computation within the task deadline. The simulation results show that the proposed multilevel scheduling method is significantly useful in ensuring the time-efficient processing of tasks and can reduce the processing delay of small tasks to a certain extent. The system integrates and optimizes, collaborates, and improves efficiency, optimizes performance management, improves employee motivation, strengthens real-time monitoring and control, provides decision support, avoids operational risks, improves enterprise competitiveness, and provides technical support for the enterprise's HRM strategy. The system is the platform for enterprise human resource management, as well as the management platform for enterprises to achieve strategic improvement and enhance enterprise competitiveness, and is a solid guarantee for enterprises to achieve takeoff.

\section{Data Availability}

The data used to support the findings of this study are available upon request to the author.

\section{Conflicts of Interest}

The author declares that there are no conflicts of interest.

\section{Acknowledgments}

This research was supported by the Philosophy and Social Sciences Planning Project of Zhejiang Province: research on Zhejiang MNE subsidiaries involvement in the host country and legitimacy acquirement mechanism from the perspective of multiple institutional logics (20NDJC346YBM).

\section{References}

[1] H.-S. Kim, H.-Y. Jeong, and H.-J. Joo, “The big data visualization technology based ecosystem cycle on high speed network," Multimedia Tools and Applications, vol. 78, no. 20, pp. 28903-28916, 2019.

[2] Z. Sun, K. Strang, and S. Firmin, "Business analytics-based enterprise information systems," Journal of Computer Information Systems, vol. 57, no. 2, pp. 169-178, 2017.

[3] W. Wang and C. Lu, "Visualization analysis of big data research based on Citespace," Soft Computing, vol. 24, no. 11, pp. 8173-8186, 2020.

[4] A. G. Finogeev, D. S. Parygin, and A. A. Finogeev, "The convergence computing model for big sensor data mining and knowledge discovery," Human-centric Computing and Information Sciences, vol. 7, no. 1, pp. 1-16, 2017.

[5] A. E. Hassanien, A. Darwish, and S. Abdelghafar, "Machine learning in telemetry data mining of space mission: basics, challenging and future directions," Artificial Intelligence Review, vol. 53, no. 5, pp. 3201-3230, 2020.

[6] M. W. Rodrigues, S. Isotani, and L. E. Zárate, "Educational data mining: a review of evaluation process in the e-learning," Telematics and Informatics, vol. 35, no. 6, pp. 1701-1717, 2018.

[7] K. Chung, H. Yoo, D. Choe, and H. Jung, "Blockchain network based topic mining process for cognitive manufacturing," Wireless Personal Communications, vol. 105, no. 2, pp. 583-597, 2019.

[8] A. De Mauro, M. Greco, M. Grimaldi, and P. Ritala, "Human resources for big data professions: a systematic classification of job roles and required skill sets," Information Processing \& Management, vol. 54, no. 5, pp. 807-817, 2018.

[9] L. Po, N. Bikakis, F. Desimoni, and G. Papastefanatos, "Linked data visualization: techniques, tools, and big data," Synthesis Lectures on the Semantic Web: Theory and Technology, vol. 10, no. 1, pp. 1-157, 2020.

[10] S. B. Sriramoju, "Opportunities and security implications of big data mining," International Journal of Research in Science and Engineering, vol. 3, no. 6, pp. 44-58, 2017.

[11] J. Lloret, M. Garcia, A. Catala, and J. J. P. C. Rodrigues, “A group-based wireless body sensors network using energy harvesting for soccer team monitoring," International Journal of Sensor Networks, vol. 21, no. 4, pp. 208-225, 2016.

[12] Y. Miao, G. Wu, and C. Liu, "Green cognitive body sensor network: architecture, energy harvesting, and smart clothing-based applications," IEEE Sensors Journal, vol. 19, no. 19, pp. 8371-8378, 2018.

[13] S. Seneviratne, Y. Hu, T. Nguyen et al., "A survey of wearable devices and challenges," IEEE Communications Surveys \& Tutorials, vol. 19, no. 4, pp. 2573-2620, 2017.

[14] J. Pagan, R. Fallahzadeh, and M. Pedram, "Toward ultra-lowpower remote health monitoring: an optimal and adaptive compressed sensing framework for activity recognition," IEEE Transactions on Mobile Computing, vol. 18, no. 3, pp. 658-673, 2018.

[15] M. Kong, J. Lin, C. H. Kang et al., "Toward self-powered and reliable visible light communication using amorphous silicon thin-film solar cells," Optics Express, vol. 27, no. 24, pp. 34542-34551, 2019.

[16] R. Punj and R. Kumar, "Technological aspects of WBANs for health monitoring: a comprehensive review," Wireless Networks, vol. 25, no. 3, pp. 1125-1157, 2019.

[17] A. Page, S. Hijazi, S. Hijazi, D. Askan, B. Kantarci, and T. Soyata, "Support systems for health monitoring using internet-of-things driven data acquisition," Services Transactions on Services Computing, vol. 4, no. 4, pp. 18-34, 2016.

[18] R. A. Reddy and N. V. Ram, "Innovative data transmission and routing strategies of wireless body area sensor networks: taxonomy, review, issues and constraints of state of the art 
contributions in recent literature," International Journal of Engineering \& Technology, vol. 7, no. 2, pp. 696-704, 2018.

[19] J. S. Olson and S. Redkar, "A survey of wearable sensor networks in health and entertainment," MOJ Applied Bionics and Biomechanics, vol. 2, no. 5, pp. 280-287, 2018.

[20] N. Belapurkar, J. Harbour, S. Shelke, and B. Aksanli, "Building data-aware and energy-efficient smart spaces," IEEE Internet of Things Journal, vol. 5, no. 6, pp. 4526-4537, 2018.

[21] H. Ghasemzadeh, R. Fallahzadeh, and R. Jafari, "A hardware-assisted energy-efficient processing model for activity recognition using wearables," ACM Transactions on Design Automation of Electronic Systems, vol. 21, no. 4, pp. 1-27, 2016.

[22] Y. A. Qadri, A. Nauman, Y. B. Zikria, A. V. Vasilakos, and S. W. Kim, "The future of healthcare internet of things: a survey of emerging technologies," IEEE Communications Surveys \& Tutorials, vol. 22, no. 2, pp. 1121-1167, 2020.

[23] M. Mohammadi, A. Al-Fuqaha, S. Sorour, and M. Guizani, "Deep learning for IoT big data and streaming analytics: a survey," IEEE Communications Surveys \& Tutorials, vol. 20, no. 4, pp. 2923-2960, 2018.

[24] L. Borzì, M. Varrecchia, G. Olmo et al., "Home monitoring of motor fluctuations in Parkinson's disease patients," Journal of Reliable Intelligent Environments, vol. 5, no. 3, pp. 145-162, 2019.

[25] G. K. Moinudeen, F. Ahmad, and D. Kumar, "IoT applications in future foreseen guided by engineered nanomaterials and printed intelligence technologies a technology review," International Journal of Internet of Things, vol. 6, no. 3, pp. 106-148, 2017.

[26] B. R. K. Mantha, C. C. Menassa, and V. R. Kamat, "A taxonomy of data types and data collection methods for building energy monitoring and performance simulation," Advances in Building Energy Research, vol. 10, no. 2, pp. 263-293, 2016.

[27] M. Chen, S. Lu, and Q. Liu, "Uniqueness of weak solutions to a Keller-Segel-Navier-Stokes model with a logistic source," Applications of Mathematics, vol. 5, 2021.

[28] A. C. Laska, A. Hellblom, and V. Murray, "The legal regulation of information and communications technologies as specific forms of a production factor for capital in the food industry," Acta Universitatis Agriculturae Et Silviculturae Mendelianae Brunensis, vol. 59, no. 5, pp. 147-156, 2014.

[29] B. Eshchanov and K. Matyakubov, "Using of information and communication technologies in the teaching of the school course in general physics," International Journal of Scientific \& Technology Research, vol. 9, no. 2, pp. 4119-4124, 2020. 